\title{
New evidence-based A1, A2, A3 alarm time zones for transferring thrombolysed patients to hyper-acute stroke units: faster is better
}

\author{
Thang S. Han ${ }^{1}$ (D) $\cdot$ Giosue Gulli ${ }^{2}$ - Brendan Affley ${ }^{2} \cdot$ David Fluck $^{3} \cdot$ Christopher H. Fry $^{4} \cdot$ Christopher Barrett $^{5}$. \\ Puneet Kakar $^{6}$ - Sapna Sharma ${ }^{1}$ • Pankaj Sharma ${ }^{1,7}$
}

Received: 31 January 2019 / Accepted: 12 April 2019 / Published online: 27 April 2019

(C) The Author(s) 2019

\begin{abstract}
Objectives The National Institute of Health and Clinical Excellence and The Royal College of Physicians recommend transferring thrombolysed patients with stroke to a hyperacute stroke unit (HASU) within $4 \mathrm{~h}$ from hospital arrival ( $\mathrm{T}_{\text {Arrival-HASU), but there is }}$ paucity of evidence to support this cut-off. We assessed if a shorter interval within this target threshold conferred a significant improvement in patient mortality.

Design We conducted a retrospective analysis of prospectively collected data from the Sentinel Stroke National Audit Programme. Setting Four major UK hyperacute stroke centres between 2014 and 2016.

Participants A total of 183 men (median age $=75$ years, IQR $=66-83$ ) and 169 women (median age $=81$ years, IQR $=72.5-88$ ) admitted with acute ischaemic stroke.

Main outcome measures We evaluated $\mathrm{T}_{\text {Arrival-HASU }}$ in relation to inpatient mortality, adjusted for age, sex, co-morbidities, stroke severity, time between procedures, time and day on arrival.

Results There were 51 (14.5\%) inpatient deaths. On ROC analysis, the AUC (area under the curve) was 61.1\% $(52.9-69.4 \%, p=$ 0.01 ) and the cut-off of $\mathrm{T}_{\text {Arrival-HASU }}$ where sensitivity equalled specificity was $2 \mathrm{~h} / 15 \mathrm{~min}$ (intermediate range $=30 \mathrm{~min}$ to $3 \mathrm{~h} /$ $15 \mathrm{~min}$ ) for predicting mortality. On logistic regression, compared with the fastest $\mathrm{T}_{\text {Arrival-HASU }}$ group within $2 \mathrm{~h} / 15 \mathrm{~min}$, the slowest $\mathrm{T}_{\text {Arrival-HASU }}$ group beyond upper limit of intermediate range ( $\geq 3 \mathrm{~h} / 15 \mathrm{~min}$ ) had an increased risk of mortality: $5.6 \%$ vs. $19.6 \%$, adjusted OR $=5.6(95 \%$ CI: $1.5-20.6, p=0.010)$.

Conclusions We propose three new alarm time zones (A1, A2 and A3) to improve stroke survival: "A1 Zone" (TArrival-HASU < $2 \mathrm{~h} / 15 \mathrm{~min}$ ) indicates that a desirable target, "A2 Zone" ( $\mathrm{T}_{\text {Arrival-HASU }}=2 \mathrm{~h} / 15 \mathrm{~min}$ to $3 \mathrm{~h} / 15 \mathrm{~min}$ ), indicates increasing risk and should not delay any further, and "A 3 Zone" ( $\mathrm{T}_{\text {Arrival-HASU }} \geq 3 \mathrm{~h} / 15 \mathrm{~min}$ ) indicates high risk and should be avoided.
\end{abstract}

Keywords Evidence-based medicine $\cdot$ ROC analysis $\cdot$ Ischaemic stroke $\cdot$ Thrombolysis $\cdot$ tPA $\cdot$ Stroke units

\section{Introduction}

Thrombolysis is an effective treatment for acute ischaemic stroke [1] and is widely performed in hyperacute stroke units

Thang S. Han

thang.han@rhul.ac.uk

1 Institute of Cardiovascular Research, Royal Holloway, University of London, TW20 0EX Egham, UK

2 Department of Stroke, Ashford and St Peter's NHS Foundation Trust, KT16 0PZ Chertsey, UK

3 Department of Cardiology, Ashford and St Peter's NHS Foundation Trust, KT16 0PZ Chertsey, UK
(HASUs) [2, 3]. Door to needle time has been a major focus of research $[4,5]$, but the time taken to transfer patients from hospital arrival to HASU is less studied and indeed may be as important. The National Institute of Health and Clinical 
Excellence (NICE) [6] and Royal College of Physicians (RCP) recommend patients presenting with an acute stroke to be transferred to a HASU within $4 \mathrm{~h}$ from hospital arrival ( $\mathrm{T}_{\text {Arrival-HASU }}$ ) [7]. This time window covers the time taken from the point at which the patient arrives hospital to brain imaging, then to intravenous thrombolysis, and followed by the transfer to HASU; thus, $\mathrm{T}_{\text {Arrival-HASU }}$ partly depends on the speed at which the patient receives brain imaging and thrombolysis.

The $\mathrm{T}_{\text {Arrival-HASU }}$ span of $4 \mathrm{~h}$ is relatively long but is related to the time needed to administer recombinant tissue plasminogen activator (rtPA). Some centres provide thrombolysis within the CT scanning unit to minimise delay while others may return the patient to the Emergency department or send to HASU for such an intervention [7].

In this study, we attempted to determine whether a more detailed subset of $\mathrm{T}_{\text {Arrival-HASU }}$ could be derived within the recommended target threshold of $4 \mathrm{~h}$ among patients undergoing thrombolysis for acute ischaemic stroke. This should provide an evidence-based target time for $\mathrm{T}_{\text {Arrival-HASU }}$ in the management of these patients.

\section{Methods}

\section{Study design, participants and setting}

We conducted a retrospective analysis of prospectively collected data from the Sentinel Stroke National Audit Programme (SSNAP) which is the national register of stroke care, comprising clinical characteristics and care quality of patients admitted to acute care hospitals in England and Wales [8]. The data were collected prospectively from the time of admission up to 6 months after stroke in patients admitted to four major UK hyperacute stroke centres in Surrey County between January 2014 and February 2016 [9, 10]. In order to test our objectives against the 4-h transfer target from the point of arrival to HASU set out by NICE [6] and RCP [7] for thrombolysed patients, the present study selected only those who were thrombolysed.

HASUs are located in each of the four study centres, providing HASU management but not endovascular services. Patients eligible for endovascular thrombolysis started intravenous thrombolysis locally and were then transferred to the regional tertiary centre, after discussion with the interventional neuroradiologists, for endovascular treatment. The SSNAP database does not collect information on patients receiving endovascular treatment in the tertiary centre. Audits at the time of data collection showed $<10 \%$ of patients were referred for endovascular treatment. Thus, patients with large artery occlusion, and not eligible for endovascular treatment, were included in this study. Because patients who had endovascular treatment were not included in this study, results refer only to patients who had intravenous treatment.

SSNAP has approval from the Confidentiality Advisory Group of the Health Research Authority to collect patient data under section 251 of the National Health Service Act 2006. No additional ethical approval was sought $[9,10]$.

\section{Socio-demographic factors and medical history}

Demographic data were collected and documented by stroke consultants and stroke nurse specialists including age at arrival, gender, past medical history (atrial fibrillation, hypertension, congestive heart failure, diabetes mellitus, previous stroke and drug history) and time points from onset of symptoms to hospital arrival, brain imaging, thrombolysis and transfer times to HASU. In addition, details of new cases of urinary tract infection and pneumonia acquired in hospital within 7 days of admission were documented.

\section{Stroke diagnosis and severity}

Stroke was diagnosed based on clinical presentation and brain imaging. The severity of stroke symptoms at arrival and $24 \mathrm{~h}$ post-thrombolysis was assessed by the National Institutes of Health for Stroke Scale (NIHSS) with a score range from no symptoms to severe stroke symptoms (NIHSS score $=0$ to 42 ).

\section{Disability and mortality}

Degree of disability or dependence with daily activities was assessed by a modified Rankin Scale (mRS), ranging from no symptoms to severe symptoms (mRS score $=0$ to 5 ) and mortality $(\mathrm{mRS}$ score $=6)$.

\section{Thrombolysis}

Thrombolysis using the fibrinolytic agent alteplase (rtPA) was considered for patients who fulfilled criteria for treatment including time from onset, confirmed diagnosis of ischaemic stroke and without contra-indications.

\section{Categorisation of variables}

Dichotomisation was applied for hypertension, congestive heart failure, atrial fibrillation, diabetes and inpatient mortality according to the presence or absence of history of the condition. Moderately severe to severe stroke on arrival or $24 \mathrm{~h}$ post-thrombolysis was defined as a NIHSS score $\geq 16$ and moderately severe to severe disability on discharge for those with $\mathrm{mRS}$ score $=4$ and 5 . 


\section{Statistical analysis}

We initially performed receiver operating characteristic (ROC) curve analysis to determine (1) the association of $\mathrm{T}_{\text {Arrival-HASU }}$ with mortality as indicated by area under the curve (AUC) and (2) the cut-offs of $\mathrm{T}_{\text {Arrival-HASU }}\left(\mathrm{d}_{0}\right)$ where sensitivity equals specificity for identifying mortality using the two-graph ROC plot technique [11, 12]; $\mathrm{d}_{0}$ was identified by interpolating from the intersection where sensitivity equals specificity $\left(\theta_{0}\right)$ and limits of intermediate range (IR) from the point where sensitivity (lower limit) and specificity (upper limit) equal 95\%. We then conducted multivariable logistic regression analysis to estimate the risk of inpatient mortality (dependent variable) from slower $T_{\text {Arrival-HASU }}$ (above the $\mathrm{d}_{0}$ and above upper limit of IR) compared with the referent group of hyper-fast $T_{\text {Arrival-HASU }}$ within $\mathrm{d}_{0}$ (predictive variables). The results were presented in four models; model 1: unadjusted, model 2: adjusted for age, sex and co-morbidities, model 3: additional adjustment to model 2 for stroke severity on arrival, time from symptom onset to hospital arrival (door time), from arrival to brain imaging and from arrival to thrombolysis (door to needle), and model 4: additional adjustment to model 3 for time of day and day of week on arrival. Results are expressed in odds ratios (OR) and 95\% confidence intervals (CI). Most variables had no missing data, which were handled in analysis using a 'listwise deletion of missing data' approach [13]. Analyses were performed using SPSS V.23.0 (SPSS Inc., Chicago, Illinois, USA). The null hypothesis was rejected when $p<0.05$.

\section{Results}

Of the 3309 patients collected in the database, $2758(83.3 \%)$ patients presented with ischaemic stroke; of the remainder 518 (15.7\%) patients had a haemorrhagic stroke and $33(1.0 \%)$ were unspecified. Among patients with ischaemic stroke, $431(15.6 \%)$ underwent thrombolysis, in whom 352 (183 men and 169 women) achieved the $\mathrm{T}_{\text {Arrival-HASU }}$ target within $4 \mathrm{~h}$; these patients were selected for analysis in relation to inpatient mortality (Fig. 1).

Median and interquartile range (IQR) age was 75 (66-83) years in men and $81(72.5-88)$ years in women. The median time from symptom onset to hospital arrival was $1 \mathrm{~h} / 9 \mathrm{~min}$ (50 $\mathrm{min}$ to $1 \mathrm{~h} / 39 \mathrm{~min}$ ), arrival to brain imaging was $19 \mathrm{~min}$ (13-28 $\mathrm{min}$ ), arrival to thrombolysis was $50 \mathrm{~min}$ (34 $\mathrm{min}$ to $1 \mathrm{~h} / 8 \mathrm{~min}$ ) and $\mathrm{T}_{\text {Arrival-HASU was }} 3 \mathrm{~h} / 00 \mathrm{~min}(2 \mathrm{~h} / 22 \mathrm{~min}$ to $3 \mathrm{~h} / 36 \mathrm{~min})$. In total, there were 51 (14.5\%) inpatient deaths (Table 1).

ROC curve analysis showed that AUC for predicting inpatient mortality was $61 \%$ (95\%CI: 53-69\%, $p=0.011$ ) (Fig. 2). The two-graph ROC plot revealed that the $\mathrm{d}_{0}$ for identifying inpatient mortality was $2 \mathrm{~h} / 15 \mathrm{~min}$ (IR=30 $\mathrm{min}$ to $3 \mathrm{~h} / 15 \mathrm{~min}$ ) (Fig. 3). Three categories of patients based on these findings were created for further assessment with inpatient mortality by logistic regression; hyper-fast group: $71(20.2 \%)$ patients with $\mathrm{T}_{\text {Arrival-HASU }}$ within $\mathrm{d}_{0}(<2 \mathrm{~h} / 15 \mathrm{~min})$, intermediate group: 143 (40.6\%) patients with $\mathrm{T}_{\text {Arrival-HASU }}$ between $\mathrm{d}_{0}$ and upper limit IR $(2 \mathrm{~h} /$ $15 \mathrm{~min}$ to $3 \mathrm{~h} / 15 \mathrm{~min}$ ), and slow group: 138 (39.2\%) patients with $\mathrm{T}_{\text {Arrival-HASU }}$ above upper limit of IR ( $\geq 3 \mathrm{~h} / 15 \mathrm{~min}$ ).

Increasing $\mathrm{T}_{\text {Arrival-HASU }}$ was associated with increasing proportions of inpatient mortality $\left(\chi^{2}=7.4, p=0.007\right)$ : hyper-fast group $=5.6 \%$, intermediate group $=14.0 \%$ and

Fig. 1 Flowchart of patient cohort

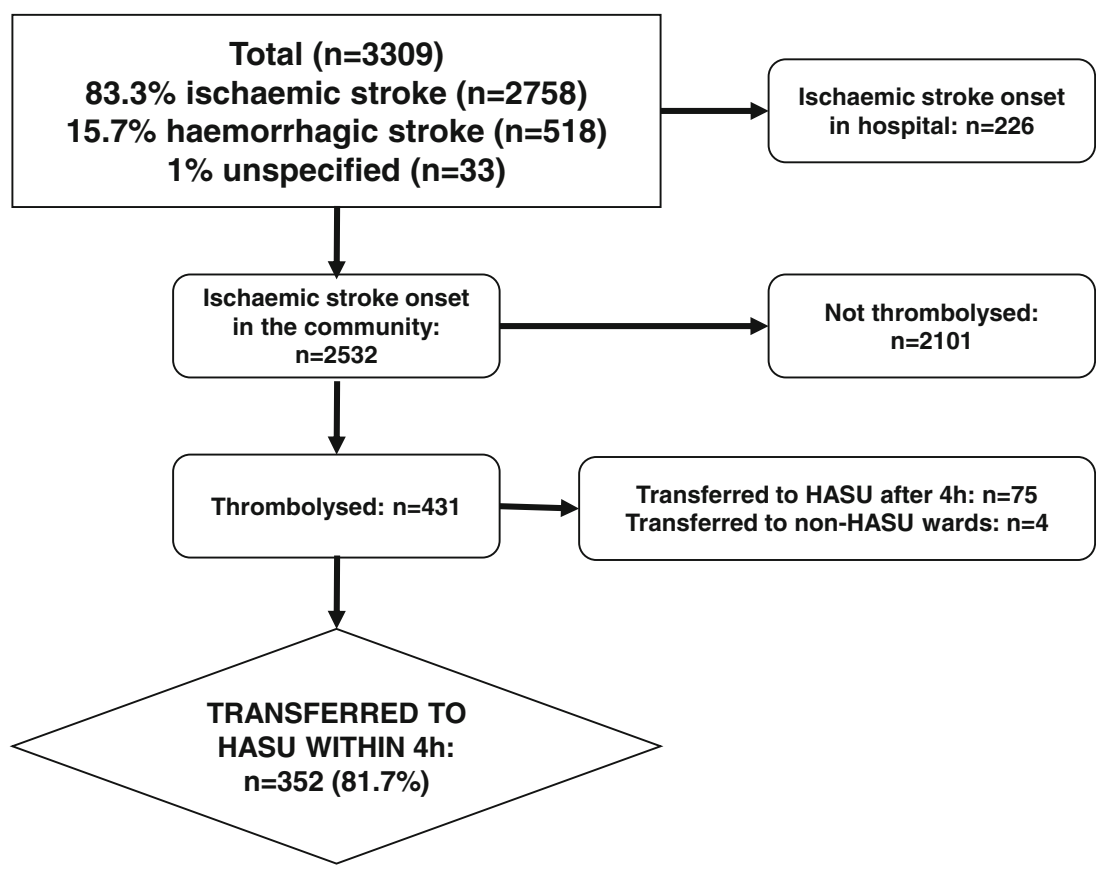


Table 1 Characteristics of 352 patients admitted with ischaemic stroke who received thrombolysis and transferred to HASU within $4 \mathrm{~h}$ on arrival

\begin{tabular}{lll}
\hline & Median & Interquartile range \\
\hline Age of men (years) & 75.0 & $66.0-83.0$ \\
Age of women (years) & 81.0 & $72.5-88.0$ \\
Time between symptom onset and arrival (h/min) & $1: 09$ & $0: 50-1: 39$ \\
Time between arrival to brain imaging (h/min) & $0: 19$ & $0: 13-0: 28$ \\
Time between arrival to thrombolysis $(\mathrm{h} / \mathrm{min})$ & $0: 50$ & $0: 34-1: 08$ \\
Time between arrival and transfer to HASU (h/min) & $3: 00$ & $2: 22-336$ \\
& $\mathrm{n}$ & Proportion $(\%)$ \\
Men/women & $183: 169$ & $52: 48$ \\
First stroke/recurrent stroke & $279: 73$ & $79.3: 20.7$ \\
Atrial fibrillation & 70 & 19.9 \\
Hypertension & 192 & 54.5 \\
Congestive heart failure & 12 & 3.4 \\
Diabetes & 55 & 15.6 \\
Inpatient mortality & 51 & 14.5 \\
\hline
\end{tabular}

slow group $=19.6 \%$. Compared with the hyper-fast group, the slow group had unadjusted OR for inpatient mortality of 4.1 (95\%CI:1.4-12.2). This OR increased to 4.9 (95\% CI:1.417.7) after adjustment for age, sex, co-morbidities, stroke severity, time from symptom onset to arrival, arrival to brain imaging and arrival to thrombolysis. Additional adjustment for time and day on hospital arrival showed the OR increased further to 5.6 (95\% CI:1.5-20.6) (Table 2). There was a trend in increasing ORs for those in the intermediate group compared with the hyper-fast group, but was not statistically significant. Extending $\mathrm{T}_{\text {Arrival-HASU }}$ for the slow group to $\geq 3 \mathrm{~h} /$ 30 min showed the risk of death elevated to 6.2-fold (95\%CI:1.7-23.1) (Table 3).

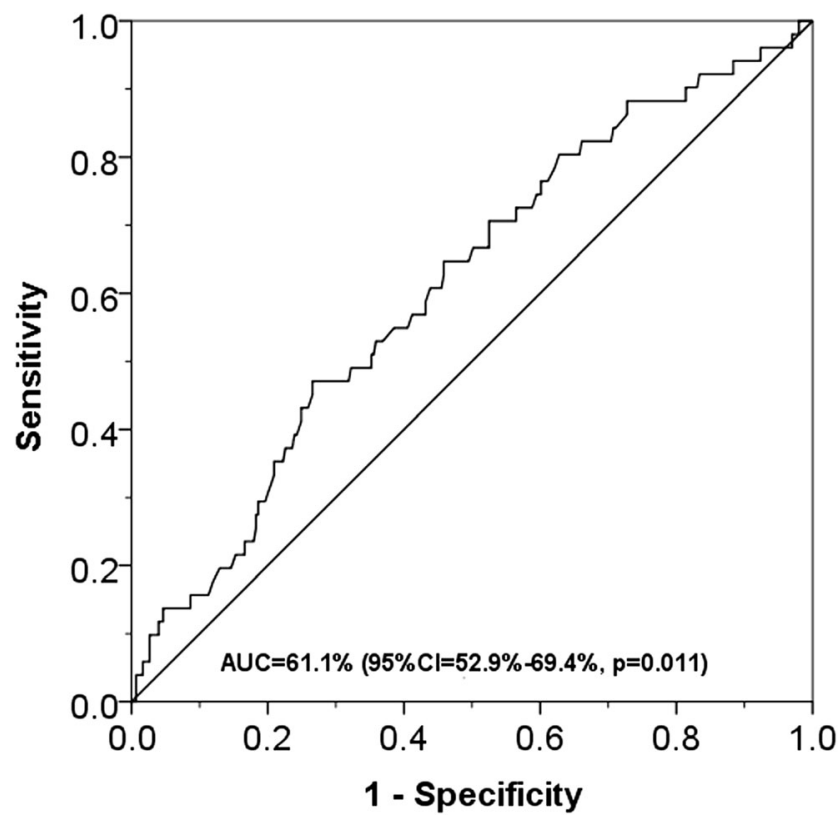

Fig. 2 ROC curve for identifying inpatient mortality from different transfer time from hospital arrival to HASU
We found that compared with the hyper-fast group, the slow group did not have increased risk (adjusted for age, sex, comorbidities, severity of stroke on arrival and time between procedures) of hospital-acquired pneumonia $(\mathrm{OR}=1.88$, 95\%CI:0.55-6.39, $p=0.315)$ or urinary tract infection $(\mathrm{OR}=$ $0.69,95 \% \mathrm{CI}: 0.22-2.19, p=0.529)$ within 7 days of admission, prolonged length of stay in HASU of $>3$ weeks $(\mathrm{OR}=1.04$, 95\%CI:0.45-2.39, $p=0.921)$ or moderately severe to severe disability on discharge (OR $=1.88,95 \% \mathrm{CI}: 0.71-4.96, p=0.203)$.

Patients presented with moderately severe to severe stroke on arrival were more likely to be thrombolysed earlier (adjusted OR $=0.24,95 \% \mathrm{CI}: 0.10-0.55)$. Faster thrombolysis on arrival resulted in reduced proportions of patients with moderately severe to severe stroke $24 \mathrm{~h}$ post-thrombolysis (i.e. proportions of patients with moderately severe to severe stroke at

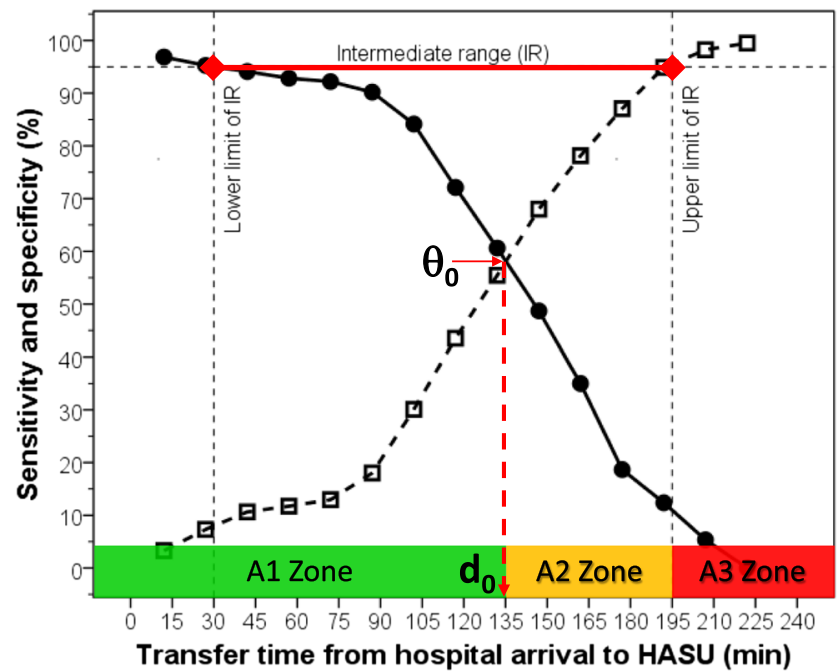

Fig. 3 Two-graph ROC plot to identify mortality showing the cut-off of $\mathrm{T}_{\text {Arrival-HASU }}\left(\mathrm{d}_{0}\right)$ interpolated from the point where sensitivity $(\bullet)$ equals specificity ( $\square$ ) $\left(\theta_{0}\right)$ and from the intermediate range (IR) at $95 \%$ level of sensitivity and specificity (red horizontal line) 
Table 2 Comparison of inpatient mortality between patients with ischaemic stroke who were transferred from arrival to HASU at different time within $4 \mathrm{~h}$ (cut-offs at $2 \mathrm{~h} / 15 \mathrm{~min}$ and $3 \mathrm{~h} / 15 \mathrm{~min}$ )

\begin{tabular}{|c|c|c|c|c|c|c|c|}
\hline & \multicolumn{7}{|c|}{ Risk of inpatient mortality } \\
\hline & $\begin{array}{l}\text { Hyper-fast group } \\
(<2 \mathrm{~h} / 15 \mathrm{~min})\end{array}$ & $\begin{array}{l}\text { Intern } \\
(\geq 21\end{array}$ & $\begin{array}{l}\text { diate group } \\
5 \text { min to } 3 \mathrm{~h}\end{array}$ & $5 \min )$ & $\begin{array}{l}\text { Slow } \\
(\geq 3\end{array}$ & $\begin{array}{l}\text { group } \\
/ 15 \mathrm{~min})\end{array}$ & \\
\hline \multirow[t]{2}{*}{ Mortality rates } & $4 / 71(5.6 \%)$ & \multicolumn{3}{|c|}{ 20/143 (14.0\%) } & \multicolumn{3}{|c|}{$27 / 138(19.6 \%)$} \\
\hline & OR & OR & $95 \% \mathrm{CI}$ & $p$ & OR & $95 \%$ CI & $p$ \\
\hline Model 1: Unadjusted & 1 & 2.72 & $0.89-8.30$ & 0.078 & 4.07 & $1.37-12.15$ & 0.012 \\
\hline $\begin{array}{l}\text { Model 2: Adjusted for age, sex, congestive heart failure, } \\
\text { hypertension, atrial fibrillation, diabetes and previous stroke }\end{array}$ & 1 & 2.94 & $0.93-9.26$ & 0.065 & 4.24 & $1.39-13.00$ & 0.011 \\
\hline $\begin{array}{l}\text { Model 3: Adjusted as in model } 2 \text { plus severity on arrival, time from } \\
\text { onset to arrival, arrival to brain imaging and arrival to thrombolysis }\end{array}$ & 1 & 3.08 & $0.87-10.91$ & 0.082 & 4.89 & $1.35-17.69$ & 0.016 \\
\hline $\begin{array}{l}\text { Model 4: Adjusted as in model } 3 \text { plus time and day on } \\
\text { hospital arrival }\end{array}$ & 1 & 3.42 & $0.95-12.32$ & 0.060 & 5.59 & $1.52-20.61$ & 0.010 \\
\hline
\end{tabular}

${ }^{\mathrm{a}}$ Referent group

$24 \mathrm{~h}$ post-thrombolysis minus proportions of patients with moderately severe to severe stroke on arrival, $p=0.003)$ : $13.6,-8.3$ and $+4.0 \%$ for tertile $1(<36 \mathrm{~min})$, tertile $2(\geq$ $36 \mathrm{~min}$ to $1 \mathrm{~h} / 19 \mathrm{~min}$ ) and tertile 3 ( $\geq 1 \mathrm{~h} / 19 \mathrm{~min}$ ), respectively.

Further analysis was conducted to assess mortality rates in different tertiles of the time between thrombolysis to HASU (i.e. eliminating the influence from the time between arrival and brain imaging and between arrival and thrombolysis). Increasing length of transfer time from thrombolysis to HASU was associated with higher proportions of inpatient mortality $\left(\chi^{2}=9.4, p=0.009\right)$ : tertile $1(<1 \mathrm{~h} / 45 \mathrm{~min})=$ $8.2 \%$, tertile $2(1 \mathrm{~h} / 45 \mathrm{~min}$ to $2 \mathrm{~h} / 30 \mathrm{~min})=13.8 \%$ and tertile $3(\geq 2 \mathrm{~h} / 30 \mathrm{~min})=22.4 \%$. Compared with the fastest transfer group, the slowest transfer group had increased risk of inpatient mortality by 3.35-fold (95\%CI:1.43-7.89, $p=$ 0.006), adjusted for age, co-morbidities and stroke severity on arrival. We found no relationship between time from arrival to brain imaging and inpatient mortality within this group of patients.

\section{Discussion}

The present study focused on the relationship between inpatient mortality and rapidity of transferring patients to HASU from hospital arrival within the recommended 4-h window among patients receiving thrombolysis. Our findings provide evidencebased cut-offs of transfer target below the currently recommended target by NICE and RCP of $4 \mathrm{~h}$, independently of demographic factors, co-morbidities, stroke severity, time to brain imaging, time to thrombolysis and time and day of admission.

We have identified two cut-off levels which provide three alarm time zones to improve stroke survival: "A1 Zone" (hyper-fast $\mathrm{T}_{\text {Arrival-HASU }}<2 \mathrm{~h} / 15 \mathrm{~min}$ ) indicates a desirable target for transferring patients to HASU as it has low mortality risk, "A2 Zone" (intermediate $\mathrm{T}_{\text {Arrival-HASU }} 2 \mathrm{~h} / 15 \mathrm{~min}$ to $3 \mathrm{~h} / 15 \mathrm{~min}$ ) indicates an increasing risk and should not delay any further, and "A3 Zone" (slow $\mathrm{T}_{\text {Arrival-HASU }} \geq 3 \mathrm{~h}: 15 \mathrm{~min}$ ) indicates high mortality risk and should be avoided. This proposal was validated by logistic regression analysis showing that compared with the

Table 3 Comparison of inpatient mortality between patients with ischaemic stroke who were transferred from arrival to HASU at different time within $4 \mathrm{~h}$ (cut-offs at $2 \mathrm{~h} / 15 \mathrm{~min}$ and $3 \mathrm{~h} / 30 \mathrm{~min}$ )

\begin{tabular}{|c|c|c|c|c|c|c|}
\hline & \multicolumn{6}{|c|}{ Risk of inpatient mortality } \\
\hline & \multirow{2}{*}{$\begin{array}{l}\begin{array}{l}\text { Hyper-fast group } \\
(<2 \mathrm{~h} / 15 \mathrm{~min})\end{array} \\
4 / 71(5.6 \%)\end{array}$} & \multicolumn{3}{|c|}{$\begin{array}{l}\text { Intermediate group } \\
(\geq 2 \mathrm{~h} / 15 \mathrm{~min} \text { to } 3 \mathrm{~h} / 30 \mathrm{~min})\end{array}$} & \multicolumn{2}{|l|}{$\begin{array}{l}\text { Slow group } \\
(\geq 3 \mathrm{~h} / 30 \mathrm{~min})\end{array}$} \\
\hline & & $25 / 17$ & $(14.5 \%)$ & & $22 / 109(20.2 \%)$ & \\
\hline Model 1: Unadjusted & 1 & 2.85 & $0.95-8.51$ & 0.061 & $4.24 \quad 1.39-12.88$ & 0.011 \\
\hline $\begin{array}{l}\text { Model 2: Adjusted for age, sex, congestive heart failure, } \\
\text { hypertension, atrial fibrillation, diabetes and previous stroke }\end{array}$ & 1 & 3.28 & $1.00-10.75$ & 0.050 & $5.46 \quad 1.63-18.31$ & 0.006 \\
\hline $\begin{array}{l}\text { Model 3: Adjusted as in model } 2 \text { plus severity on arrival, time from } \\
\text { onset to arrival, arrival to brain imaging and arrival to thrombolysis }\end{array}$ & 1 & 3.67 & $1.08-12.46$ & 0.037 & $6.38 \quad 1.78-22.86$ & 0.004 \\
\hline $\begin{array}{l}\text { Model 4: Adjusted as in model } 3 \text { plus time and day on } \\
\text { hospital arrival }\end{array}$ & 1 & 4.03 & $1.15-14.19$ & 0.030 & $6.24 \quad 1.68-23.12$ & 0.006 \\
\hline
\end{tabular}

${ }^{\mathrm{a}}$ Referent group 
hyper-fast group in the "A1 Zone", the slow group in the "A3 Zone" had a 5.6-fold greater adjusted risk of inpatient mortality. We found that among the 138 patients (39.2\% of the study sample) in the "A3 Zone", there were 27 deaths $(52.9 \%$ of total mortality), compared with only 4 deaths $(7.8 \%)$ among the 71 patients in the "A1 Zone". Our findings therefore provide strong support for the use of these time zones.

There exists a number of service standards set by authoritative bodies including NICE [6] and RCP [7] with respect to the acute stroke pathway; these include the time from symptom onset to hospital admission, followed by brain imaging, thrombolysis and transfer to HASU. The recommended 4-h window has been adopted for number of years without modification despite rapid advances in hyperacute stroke service have been achieved in recent years [3]. Brain imaging is now operating continuously $24 \mathrm{~h}$ every day with the proportions of patients receiving CT scan within $1 \mathrm{~h}$ of hospital arrival rose from $41 \%$ in 2013 to $51 \%$ in 2016 [8]. Over this 3-year period, "door to needle" time has progressively been quickened by $2 \min 20 \mathrm{~s}$ a year [8]. Integrated network linking the community and specialist stroke centres has been firmly established [2]. National audits such as SSNAP provide continuous updated stroke service performance and recommendations [8]. The 4-h $\mathrm{T}_{\text {Arrival-HASU }}$ target therefore may be outdated and a new evidence-based cut-off should now be considered. Further studies are suggested to examine other standards such as time to transfer from the point of thrombolysis to HASU to provide an optimal evidence-based target. The present study did not examine those factors that influence actual $\mathrm{T}_{\text {Arrival-HASU }}$ times and this is the next step that are practical at both local and regional/national levels. Examples can include, minimising out-of-hours and weekend effects by ensuring that the right capacity remains available to allow seamless flow of patients through the pathway to increase patient throughput and avoid delayed transfer of care.

Additional analysis found that rapid $\mathrm{T}_{\text {Arrival-HASU }}$ did not significantly associate with a number of adverse outcomes (nosocomial infections within 7 days of admission, prolonged hospital stay or disability on discharge) suggesting that early transfer to HASU not only lowers the rates of mortality but also has no other detrimental effects to the patients. Thus, rapid transfer to HASU provides favourable outcomes to patients treated with thrombolysis for acute stroke. This may in part be explained by a number of benefits gained from faster transfer to HASU including early review from hyperacute stroke specialists and supportive care such as dysphagia screen and appropriate nutrition, which has been shown to associate with better outcomes [9]. We have calculated that dysphagia screen in hyper-fast group was performed within $4 \mathrm{~h}$ of admission in $97.2 \%$, between 4 and $72 \mathrm{~h}$ in $2.8 \%$ and $0 \%$ beyond $72 \mathrm{~h}$; in comparison, the corresponding figures for the slow group were $89.9 \%, 8.0 \%$ and $2.2 \%\left(\chi^{2}=12.6, p=\right.$ 0.007 ). It should be borne in mind, however, that establishing an 'optimal' $\mathrm{T}_{\text {Arrival-HASU }}$ requires consideration of a number of factors including stability of the patients and trade-offs between benefits and risks in terms of financial costs to accommodate HASU service and safety of the patients when varying the desired true positive rate (sensitivity) and against false positive rate (1-specificity).

Evidence from previous studies has indicated that the earlier is thrombolysis, the greater the likelihood for good stroke outcomes $[14,15]$. However, little is known about how stroke severity influences the likelihood of receiving interventions [16]. In this study, we observed that patients with more severe stroke on arrival, indicated by NIHSS score, were more likely to be thrombolysed at earlier times, although they were not necessarily transferred to HASU earlier. Time to stabilise the patient or availability of HASU beds may in part explain this discrepancy. Previous analysis of SSNAP data has found that admission to HASU within $4 \mathrm{~h}$ varied with days of the week and there was a variation in mortality with time of the day but this association was not apparent between days of the week in which patients were admitted [17, 18]. Our study found that adjustment for time and day on arrival slightly accentuated the relationship between $\mathrm{T}_{\text {Arrival-HASU }}$ and mortality, as reflected by an increase in the OR.

\section{Strengths and limitations}

The strengths in the present study lie in their completeness of data including a number of variables, such as time to brain imaging and thrombolysis, which could potentially confound the associations between $\mathrm{T}_{\text {Arrival-HASU }}$ and mortality. Various cut-offs of $\mathrm{T}_{\text {Arrival-HASU }}$ could be derived by the powerful two-graph ROC analysis [11, 12] for further examination by logistic regression. Our conclusions are robustly supported by logistic regression analysis where potential confounders were simultaneously adjusted using multivariate modelling as well as further analysis from the point of thrombolysis to HASU. This eliminates the variable periods prior to thrombolysis such time from hospital arrival to brain imaging or from arrival to thrombolysis. We have also extended our analysis to include patients who did not meet the 4-h target of transfer from hospital arrival to HASU and showed that the risk of mortality were similarly elevated among the slowest transfer group. We also explored other cut-offs for $\mathrm{T}_{\text {Arrival-HASU }}$ including the use of tertiles or limits of IR where sensitivity (lower limit) and specificity (upper limit) were at 90\% and found similar, but weaker, patterns in the prediction of inpatient mortality. The present study is limited by its subjects who were restricted to four centres. However, this study population has similar characteristics to those in England and Wales [8] but caution should, however, be taken to extrapolate our findings. The overall mortality rate among patients treated with thrombolysis in the present study (including those who were transferred to HASU beyond $4 \mathrm{~h}$ ) was $14.8 \%$ (64/431) which is lower than the reported figure of $19.4 \%$ from a recent meta-analysis of 27 studies [19]. We focused on " $T_{\text {Arrival-HASU", which is as relevant as }}$ "door to needle time" or "thrombolysis to HASU" time. It is 
likely that the outcomes would be similar for these three measures as their journeys are dependent on each other. The reason that we selected $\mathrm{T}_{\text {Arrival-HASU }}$ is that there is a clear recommended 4-h target transfer window set out by NICE [6] and RCP [7] with which we could compare.

Another limitation of the present study is that, due to the inherent design of SSNAP, other features were not collected that could be associated with clinical outcome in thrombolysed patients, e.g. altered level of consciousness, symptomatic intracranial haemorrhage, presence of large vessel occlusion and site of occlusion. In addition, there is a lack of information about hyperacute complications such as severe hypertension, acute angioedema, hyperacute haemorrhage or subtypes of ischaemic stroke that would be useful for a better understanding of the reasons for increased $\mathrm{T}_{\text {Arrival-HASU} \text {. }}$

In conclusion, $\mathrm{T}_{\text {Arrival-HASU }}$ below the recommended target window of $4 \mathrm{~h}$ associates with lower inpatient mortality. We propose three new alarm time zones to improve stroke survival: "A1 Zone" ( $\left.\mathrm{T}_{\text {Arrival-HASU }}<2 \mathrm{~h} / 15 \mathrm{~min}\right)$ indicates a desirable target for transferring patients to HASU, "A2 Zone" $\left(\mathrm{T}_{\text {Arrival- }}\right.$ $\mathrm{HASU}=2 \mathrm{~h} / 15 \mathrm{~min}$ to $3 \mathrm{~h} / 15 \mathrm{~min}$ ) indicates an increasing risk and should not delay any further, and "A3 Zone" $\left(\mathrm{T}_{\text {Arrival- }}\right.$ HASU $\geq 3 \mathrm{~h} / 15 \mathrm{~min}$ ) indicates high risk and should be avoided.

Acknowledgements The authors wish to thank patients and all those who were involved in the surveys.

\section{Compliance with ethical standards}

Conflict of interests The authors declare that they have no conflicts of interest.

Contributor and guarantor information TSH and PS reviewed the topic related literature and performed the study concept and analysis design. BA, GG, CB and PK performed the study coordination and data collection. TSH wrote the first draft, analysed, interpreted the data and revised the manuscript. CHF, DF, SS and PS edited the manuscript. All authors checked, interpreted results and approved the final version.

Provenance and peer review Not commissioned; externally peer reviewed.

Data sharing statement No additional data are available.

Open Access This article is distributed under the terms of the Creative Commons Attribution 4.0 International License (http:// creativecommons.org/licenses/by/4.0/), which permits unrestricted use, distribution, and reproduction in any medium, provided you give appropriate credit to the original author(s) and the source, provide a link to the Creative Commons license, and indicate if changes were made.

\section{References}

1. Wahlgren N, Ahmed N, Dávalos A et al (2007) Thrombolysis with alteplase for acute ischaemic stroke in the Safe Implementation of
Thrombolysis in Stroke-Monitoring Study (SITS-MOST): an observational study. Lancet 369:275-282

2. Liu SD, Rudd A, Davie C (2011) Hyper acute stroke unit services. Clin Med 11:213-214

3. Morris S, Hunter RM, Ramsay AI et al (2014) Impact of centralising acute stroke services in English metropolitan areas on mortality and length of hospital stay: difference-in-differences analysis. BMJ 349:g4757

4. Fonarow GC, Smith EE, Saver JL et al (2011) Improving door-toneedle times in acute ischemic stroke: the design and rationale for the American Heart Association/American Stroke Association's Target: Stroke initiative. Stroke 42:2983-2989

5. Saver JL, Fonarow GC, Smith EE et al (2013) Time to treatment with intravenous tissue plasminogen activator and outcome from acute ischemic stroke. JAMA 309(23):2480-2488

6. NICE. https://www.nice.org.uk/guidance/qs2/chapter/qualitystatement-1-prompt-admission-to-specialist-acute-stroke-units.

7. Royal College of Physicians. Prepared by Intercollegiate Stroke Working Party. National clinical guideline for stroke. Fifth Edition 2016. https://www.strokeaudit.org/SupportFiles/Documents/ Guidelines/2016-National-Clinical-Guideline-for-Stroke-5t-(1).aspx

8. Royal College of Physicians. Clinical effectiveness and evaluation unit on behalf of the intercollegiate stroke working party. SSNAP JanuaryMarch 2016. Public Report. https://www.strokeaudit.org/Documents/ National/AcuteOrg/2016/2016-AOANationalReport.aspx

9. Han TS, Lean ME, Fluck D et al (2018) Impact of delay in early swallow screening on pneumonia, length of stay in hospital, disability and mortality in acute stroke patients. Eur J Clin Nutr. https:// doi.org/10.1038/s41430-018-0148-4

10. Han TS, Fry CH, Fluck D et al (2018) Anticoagulation therapy in patients with stroke and atrial fibrillation: a registry-based study of acute stroke care in Surrey, UK. BMJ Open 8(7):e022558

11. Greiner M, Sohr D, Göbel P (1995) A modified ROC analysis for the selection of cut-off values and the definition of intermediate results of serodiagnostic tests. J Immunol Methods 185(1):123-132

12. Han TS, Van Leer EM, Seidell JC, Lean ME (1996) Waist circumference as a screening tool for cardiovascular risk factors: evaluation of receiver operating characteristics (ROC). Obes Res 4:533-547

13. Acock AC (2005) Working with missing values. J Marriage Fam 67:1012-1028

14. Emberson J, Lees KR, Lyden P et al (2014) Effect of treatment delay, age, and stroke severity on the effects of intravenous thrombolysis with alteplase for acute ischaemic stroke: a meta-analysis of individual patient data from randomised trials. Lancet 384:1929-1935

15. Meretoja A, Keshtkaran M, Saver JL et al (2014) Stroke thrombolysis: save a minute, save a day. Stroke 45:1053-1058

16. Ramsay AI, Morris S, Hoffman A et al (2015) Effects of centralising acute stroke services on stroke care provision in two large metropolitan areas in England. Stroke 46:2244-2251

17. Bray BD, Ayis S, Campbell J et al (2014) Associations between stroke mortality and weekend working by stroke specialist physicians and registered nurses: prospective multicentre cohort study. PLoS Med 11(8):e1001705

18. Bray BD, Cloud GC, James MA et al (2016) SSNAP collaboration. Weekly variation in health-care quality by day and time of admission: a nationwide, registry-based, prospective cohort study of acute stroke care. Lancet 388:170-177

19. Wardlaw JM, Murray V, Berge E, del Zoppo GJ (2014) Thrombolysis for acute ischaemic stroke. Cochrane Database Syst Rev 7:109-110

Publisher's note Springer Nature remains neutral with regard to jurisdictional claims in published maps and institutional affiliations. 